\title{
RESPUESTA EN PRODUCCIÓN DE LECHE RELACIONADA AL NIVEL DE INCLUSIÓN DE ALIMENTO CONCENTRADO EN LA DIETA DE VACAS LECHERAS DE FINCAS ASOCIADAS A PRODUCTORES DE MONTEVERDE S.A.
}

Alejandro Saborío-Montero, Randall Arguedas-Sánchez², Juan José Monge-Maroto ${ }^{3}$

\section{RESUMEN}

El objetivo de esta investigación fue evaluar la respuesta productiva relacionada al nivel de inclusión de alimento concentrado en la dieta de vacas lecheras en fincas, asociadas a la empresa Productores de Monteverde S.A. La recolección de información se llevó a cabo durante el cuarto trimestre del año 2007 y el primer trimestre del 2008, en fincas ubicadas en: Puntarenas, Guanacaste y Alajuela, Costa Rica. Un total de 75 fincas con animales en pastoreo fueron incluidas en el estudio para estimar: la dosis de alimento concentrado promedio utilizada diariamente por animal, la productividad diaria promedio por animal y la respuesta productiva asociada a la dosificación de alimento concentrado. Un análisis de regresión lineal determinó que las vacas produjeron 5,52 kg de leche a base del forraje consumido y reservas corporales y por cada kilogramo de alimento concentrado dosificado en la ración, las vacas produjeron 1,67 kg de leche adicional (1,92 $\mathrm{kg}$ leche/kg concentrado en base seca, $\left.R^{2}=0,71\right)$. La producción de leche fue significativamente mayor $(P<0,05)$, al incrementar la oferta de alimento concentrado para tres distintas categorías de dosificación ( $<3 \mathrm{~kg}, 3$ a $6 \mathrm{~kg},>6 \mathrm{~kg}$ ). La asociación entre la oferta de alimento concentrado por animal por día y la proporción de sólidos totales producidos en la leche fue baja $\left(R^{2}=0,1\right)$. En condiciones similares a las del estudio, se considera que el alimento concentrado es un insumo indispensable para aumentar la rentabilidad delos sistemas productivos lecheros.

Palabras clave: Producción de leche, alimento concentrado, vacas lecheras, pastoreo.

\footnotetext{
${ }^{1}$ Universidad de Costa Rica. Escuela de Zootecnia y Centro de Investigación en Nutrición Animal. San José, Costa Rica. Autor para correspondencia: alejandro.saboriomontero@ucr.ac.cr

${ }^{2}$ Planta de Alimentos Balanceados, Cooperativa de Productores de Leche Dos Pinos, Alajuela, Costa Rica. ${ }^{3}$ Gerencia de Relaciones Ganaderas, Sigma Alimentos, Costa Rica.

Recibido: 25 agosto $2015 \quad$ Aceptado: 11 setiembre 2015
} 


\section{ABSTRACT}

Response in milk yield related to the level of inclusion of concentrate feed in the diet of dairy cows in farms associated to Productores de Monteverde S.A. The aim of this research was to evaluate the response in milk yield associated with the level of inclusion of concentrate feeds in the diet of dairy cows in farms associated to the company Productores de Monteverde S.A. Data was collected during the fourth quarter of 2007 and first quarter of 2008 in farms situated in Puntarenas, Guanacaste and Alajuela, Costa Rica. A total of 75 grazing dairy farms were included in the study to estimate: the dose of concentrated feed used daily by animal, the costs associated with the purchase of concentrate feed, daily average productivity per cow and the milk yield response associated with the dosage of concentrate feed. A linear regression analysis determined that the cows yielded $5.52 \mathrm{~kg}$ of milk based on consumed forage and body reserves, and per each kilogram of concentrate dosed in the diet, cows yielded $1.67 \mathrm{~kg}$ of additional milk(1,92 kg milk/kg concentrate, Dry Matter Basis, $\left.R^{2}=0.71\right)$. Milk yield was significantly higher $(P<0.05)$, with increased the offer of concentrate feed for three different categories of dosage ( $<3 \mathrm{~kg}$, 3 to $6 \mathrm{~kg},>6 \mathrm{~kg}$ ). The association between offer of concentrate feed per animal per day and the proportion of total milk solids produced was low $\left(R^{2}=0.1\right)$. Under conditions similar to this study, it is considered that concentrate feed is an indispensable input to increase the profitability of the dairy production system.

Keywords: Milk yield, concentrate feed, dairy cows, grazing. 


\section{INTRODUCCIÓN}

Los animales rumiantes tienen un tracto gastrointestinal con características anatómicas adaptadas para alimentarse a base de forraje y realizar un proceso fermentativo utilizando como materia prima este tipo de alimentos.

Los ácidos grasos volátiles (AGV) son los productos de la fermentación ruminal cuantitativamente más importantes (Storm, et al. 2012). La inclusión de alimentos concentrados en la dieta de los rumiantes incrementa la producción total de AGV y altera las proporciones de éstos. Particularmente la concentración de propionato se favorece en relación a los otros AGV (Rojas, 1995; Sutton, et al. 2003). En los sistemas de lechería bovina especializada, el uso de alimento concentrado ha sido una estrategia que ha implementado el ser humano para mejorar el rendimiento productivo de las vacas.

El mecanismo mediante el cual estos AGV son absorbidos no ha sido completamente elucidado, sin embargo la teoría más aceptada propone la difusión pasiva de AGV no ionizados a través del epitelio ruminal (Storm, et al. 2012).

Se estima que entre 50 a $85 \%$ de los AGV son directamente absorbidos a través de la pared retículo-ruminal y 15 a $50 \%$ pasa hacia partes distales del sistema digestivo (Aschenbach, et al. 2010). El paso de los AGV hacia la sangre ocurre mediante la remoción de estos ácidos de la parte serosa del epitelio retículo-ruminal hacia el torrente sanguíneo (Storm y Kristensen, 2010).

Estos productos finales de la fermentación, proveen a la vaca con la mayor parte de los precursores energéticos para los procesos metabólicos (Storm et al., 2012). Uno de estos procesos consiste en la síntesis de leche.

El aumento en la rentabilidad de las lecherías es una de las metas más importantes para los productores, técnicos y profesionales involucrados en el sector lácteo. El análisis de los factores que influencian la optimización del ingreso económico de la empresa lechera es importante para procurar la sostenibilidad de estos sistemas productivos.

En la continua búsqueda para aumentar la rentabilidad de las lecherías es necesario determinar cuáles insumos son indispensables desde un punto de vista técnico y 
económico. En algunas ocasiones se toman decisiones para reducir costos, con el objetivo de ampliar el margen de ganancia.

Cuando esas decisiones están basadas en criterios arbitrarios, esto puede implicar una afectación de la productividad y viabilidad económica del sistema. La reducción de un insumo beneficioso, como lo es el alimento concentrado, puede limitar la eficiencia productiva de la finca, debido al limitado aporte energético de los forrajes tropicales (Sánchez y Soto, 1999), reduciendo los ingresos netos.

El presente documento pretende caracterizar la respuesta en producción láctea y su impacto económico, en función a diferentes niveles de inclusión de alimentos concentrados en la dieta de vacas lecheras, a partir de información recopilada de75 fincas asociadas a la empresa Productores de Monteverde S.A. en diferentes zonas de Puntarenas, Guanacaste y Alajuela, Costa Rica.

\section{MATERIALES Y MÉTODOS}

Un total de 75 fincas lecheras asociadas a la empresa Productores de Monteverde S.A. fueron seleccionadas para que sus encargados completaran una encuesta en la cual se les consultaba sobre información para caracterizar la finca, así como las compras semanales de alimento concentrado y la producción de leche. Se verificó la información mediante documento físico (facturas, recibos de entrega de leche, libros de registros) 0 digital (registros digitados en programa VAMPP Bovino (Noordhuizen y Buurman, 1984)) en los casos en que fue posible.

Mediante el uso de los archivos de calidad de leche de la empresa Productores de Monteverde S.A., se extrajo información referente al porcentaje de sólidos totales promedio de la leche producida para el mes de marzo de 2008 en 70 de las 75 fincas en estudio, la información de sólidos totales en la leche de 5 fincas no pudo ser extraída. El único criterio de inclusión fue que la finca estuviese asociada a dicha empresa. Se

incluyeron fincas lecheras pertenecientes a las provincias de Puntarenas, Guanacaste y Alajuela. 
La finca promedio típicamente medía entre 5 y 15 hectáreas, utilizaba entre 25 y 30 apartos para pastoreo, con un sistema de rotación a tiempo fijo y un día de ocupación por aparto. Los animales en la mayoría de las fincas se alimentaban exclusivamente de pasto estrella africana (Cynodon nlemfuensis) y alimento concentrado (87\% materia seca, 16\% proteína cruda, $3350 \mathrm{Kcal}$ ED $\left.\left(1,785 \mathrm{EN}_{\mathrm{L}}\right)\right)$. Las razas más utilizadas en las fincas de estudio fueron: Holstein, Jersey, y sus cruces.

Se evaluó la información de producción de leche y dosificación de alimento concentrado mediante un análisis de correlación de Pearson y un análisis de regresión lineal. Para evaluar la relación entre los sólidos totales y el uso de alimento concentrado se realizó un análisis de regresión cuadrática.

Para comparar diferentes categorías de oferta de alimento concentrado, se efectuó una prueba estadística de comparación múltiple de Tukey-Cramer, protegida por un análisis de varianza, además se ajustó la producción obtenida a base de pasto y reservas corporales en función a animales de primero, segundo, y tres o más partos. Asumiendo una distribución equitativa según parición.

Los datos se consideraron estadísticamente significativos cuando $P<0,05$. Estos se tabularon y analizaron haciendo uso de los programas de cómputo Microsoft Excel ${ }^{\circledR} \mathrm{e}$ Infostat 1.0. (Di Rienzo, et al. 2011). 


\section{RESULTADOS Y DISCUSIÓN}

Según los datos de la encuesta, la estructura promedio de hato de estas fincas consistía en: 19 vacas en ordeño, 9 vacas secas, 4 vacas prontas a parir, 8 novillas y 8 terneras. Las vacas en producción se suplementaban en promedio con 3,79 $\mathrm{kg}$ de alimento concentrado vaca/día. Cada vaca producía en promedio 11,83 kg de leche/día

\section{Producción de leche}

El análisis de correlación entre las variables de consumo de alimento concentrado promedio por vaca y producción láctea promedio por vaca para las 75 fincas arrojó un coeficiente de correlación de 0,84 $(P<0,0001)$. El coeficiente de correlación $(r)$ es un estadístico que mide la fuerza de la relación lineal entre dos variables (Hoshmand, 1994).

La respuesta en producción de leche obtenida en función de la cantidad de alimento concentrado agregado se describe en la Figura 1.La ecuación [1] de la Figura 1A indica que cuando el valor del eje $X$ ( $k g$ de concentrado) fue cero, el valor del eje $Y$ ( $k g$ de leche) fue 5,5166. En otras palabras, cuando los animales del estudio no consumieron concentrado, produjeron en promedio y según reglas de redondeo habituales, $5,52 \mathrm{~kg}$ de leche a base de pasto y reservas corporales.
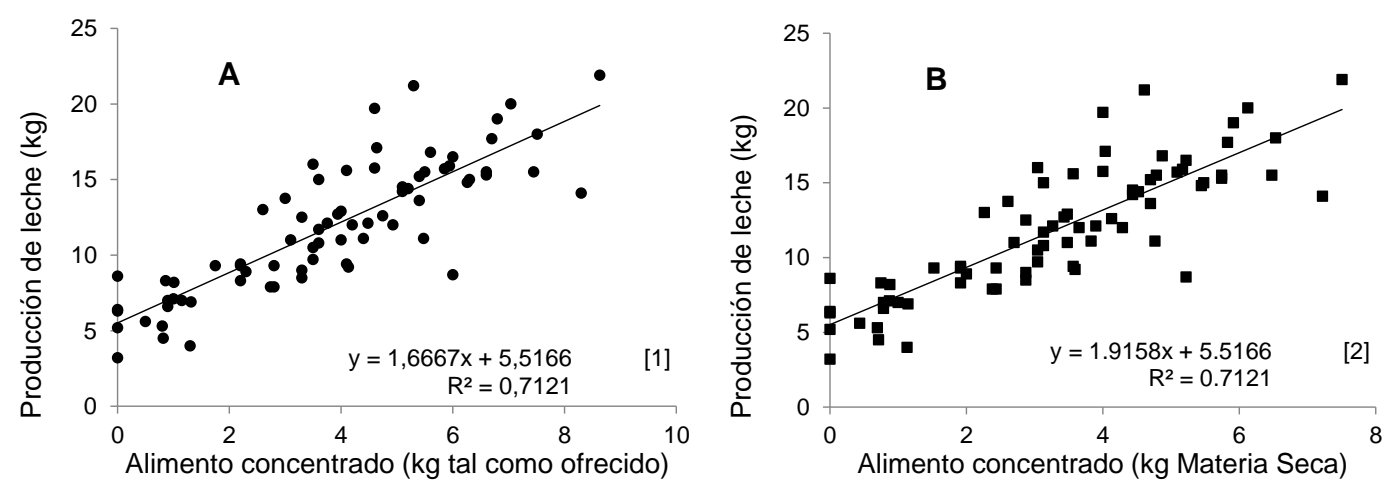

Figura 1. Efecto de la oferta de alimento concentrado tal como ofrecido $(A)$ y en materia seca (B) sobre la producción de leche en 75 fincas de productores asociados a Productores de Monteverde S.A. 
La estimación de producción se realizó en todos los animales productivos sin estratificar según número de lactancia. Sin embargo según Meyeres, et al. (2004) los animales de primer parto producen $83 \%$ del potencial productivo adulto (P. A.) y los de segundo parto, el 94\% de dicho potencial. Asumiendo una proporción igual para animales de primero, segundo y tres o más partos dentro de los hatos, la estimación del potencial productivo a base de pasto y reservas corporales se puede ajustar por número de parto según la ecuación [3]:

$$
\left(\left(P . A .{ }^{*} 0,83\right)+\left(\text { P. A. }{ }^{*} 0,94\right)+\left(\text { P. A. }{ }^{*} 1\right)\right) / 3=5,52 \mathrm{~kg}
$$

Al despejar esta ecuación para vacas adultas, animales de $2^{\circ}$ parto y animales de $1^{\circ}$ parto se obtienen respectivamente producciones de 5,98; 5,62y 4,96 kg de leche a base de pasto y reservas corporales.

De la ecuación [1] (Figura 1A), también se puede extraer que por cada kg de alimento concentrado ofertado (tal como ofrecido), se produjeron 1,6667 $\approx 1,67 \mathrm{~kg}$ de leche. Si se estima la respuesta productiva en relación al alimento concentrado en base seca (ecuación [2], Figura 1B), se obtiene una producción de 1,92 kilogramos de leche por cada kilogramo de materia seca de alimento concentrado.

Esta respuesta en producción de leche es superior a la reportada en una revisión sobre el tema (Bargo, et al. 2003) en donde el promedio general de respuesta productiva para varios estudios (Bargo, et al. 2002; Delaby, et al. 2001; Gibb, et al. 2002; Reis y Combs, 2000; Sayers, 1999; Robaina, et al. 1998; Walker, et al. 2001) fue $1 \mathrm{~kg}$ de leche/kg de alimento concentrado (base seca).

El $R^{2}$, también llamado coeficiente de determinación, representa la proporción de la variabilidad total explicada por el modelo de regresión lineal (Kaps y Lamberson, 2009). De igual forma este estadístico provee información de que tan bien se ajusta la línea de mínimos cuadrados (también conocida como línea de tendencia o línea de ajuste) a los datos (Hoshmand, 1994). En el caso del $R^{2}$ asociado a la ecuación de regresión lineal evaluada [1], éste indicó que aproximadamente el $71 \%\left(R^{2}=0,7121, P<0,0001\right)$ de la variabilidad de la producción de leche se explicó por la relación lineal entre el uso del 
alimento concentrado y la producción láctea en la población de estudio. El valor asociado al coeficiente de determinación en este estudio se considera alto.

Existen pruebas de comparación múltiple que permiten determinar la existencia de diferencias estadísticamente significativas $(P<0,05)$ entre medias de variables de interés, según categorías, estratos o tratamientos. Una de ellas es la prueba de Tukey-Cramer, la cual puede ser utilizada cuando los tamaños de muestra son desiguales (Balzarini, et al. 2008). Al estratificar la oferta de alimento concentrado de la población en estudio en tres categorías ( $<3 \mathrm{~kg}$, 3 a $6 \mathrm{~kg},>6 \mathrm{~kg}$ ), y realizar la prueba de comparación múltiple descrita, se obtuvo diferencias significativas $(P<0,05)$ para las medias de producción de leche de los tres estratos (Figura 2).

Las fincas que ofertaron más alimento concentrado, obtuvieron mayor producción de leche promedio de sus animales, posiblemente por una mayor disponibilidad de energía en la dieta total. Según investigaciones previas (Villalobos y Arce, 2014) realizadas en fincas asociadas a Productores de Monteverde S. A., algunas de las cuales están incluidas en el presente estudio, el contenido proteico no es limitante para satisfacer los requerimientos del ganado lechero de la zona.

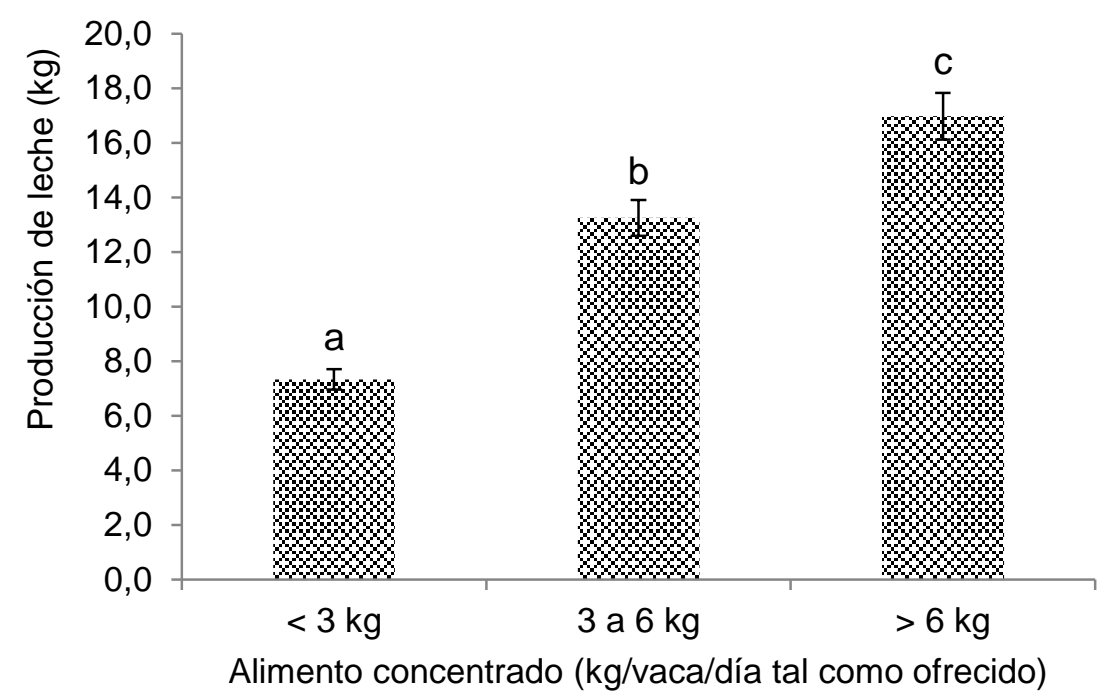

Figura 2. Producción de leche según estratos de oferta de alimento concentrado en 75 fincas de productores asociados a Productores de Monteverde S.A. Letras distintas entre columnas indican diferencias significativas $(P<0,05)$ según la prueba de Tukey-Cramer. 
La energía neta de lactancia $\left(E N_{L}\right)$ se define como la energía contenida en la leche producida (NRC, 2001) y ésta puede ser calculada a partir del porcentaje de grasa en leche según la ecuación [4] propuesta por Tyrrell y Reid (1965).

$$
\mathrm{EN}_{\mathrm{L}}(\mathrm{Mcal} / \mathrm{kg} \text { de leche })=0,360+[0,0969(\% \text { Grasa })]
$$

Los valores típicos de grasa láctea en las fincas de estudio oscilaban entre 3,5 y 4,5\% por lo que según la ecuación [4], la $\mathrm{EN}_{\mathrm{L}}$ del alimento concentrado necesaria para producir un kilogramo de leche oscilaría entre 0,70 y 0,80 , esto quiere decir que con alimentos concentrados cuya $\mathrm{EN}_{\mathrm{L}}$ fue 1,785 Mcal se podrían producir 2,6 kg de leche con 3,5\% de grasa, o 2,2 kg de leche con 4,5\% de grasa por cada kilogramo de alimento concentrado adicional consumido por el animal. Sin embargo esa relación descrita en la literatura difirió de lo observado en este estudio, en el cual, bajo las condiciones específicas de las fincas, y según el rendimiento productivo estimado en base a alimento concentrado (1,92 kg leche/kg MS conc.), se obtuvo un valor necesario de consumo energético de 0,93Mcal $E N_{\mathrm{L}}$ para producir un $\mathrm{kg}$ de leche en base a alimento concentrado con 1,785 Mcal $E N_{\mathrm{L}}$.

Entre las causas probables que podrían aumentar la proporción de energía necesaria para producir un kilogramo de leche para las vacas en pastoreo en relación a las estabuladas (con las cuales se desarrolló la ecuación [4]), se encuentran: distancia que caminan los animales desde el aparto de pastoreo hasta la sala de ordeño, actividad de pastoreo y topografía de la finca donde pastorean las vacas.

Según el ARC (1980), asumiendo que una vaca se ordeña 2 veces al día, pesa $450 \mathrm{~kg}$ y pastorea en topografía montañosa, ubicada a 250 metros de distancia del lugar de ordeño, el gasto energético diario estimado para este animal se incrementa en 0,20 Mcal $\left(E N_{L}\right)$ por la distancia caminada, 0,54 Mcal $\left(E N_{L}\right)$ por la actividad de pastoreo y 1,35 Mcal $\left(E N_{\mathrm{L}}\right.$ ) por la topografía ( $25 \mathrm{~m}$ de pendiente caminados 4 veces al día) para un total de 2,09 Mcal $\left(E N_{L}\right)$ de incremento por sobre una vaca estabulada. 
Tomando en cuenta el escenario descrito anteriormente, para el promedio de producción observado de $11,83 \mathrm{~kg}$ de leche, al realizar el ajuste por distancia caminada, actividad de pastoreo y topografía, la energía necesaria para producir un litro de leche a base de alimento concentrado sería $0,75 \mathrm{Mcal} E \mathrm{~N}_{\mathrm{L}}$. Este valor energético coincide con los valores esperados según la ecuación [4].

Estas diferencias entre características ambientales de los diferentes sistemas pecuarios deben ser evaluadas para obtener mejores resultados productivos (Saborío-Montero, 2010).

\section{Sólidos lácteos}

Es importante destacar que el comportamiento productivo lineal observado no es sostenible si se suplementan cantidades excesivas de alimento concentrado, que sobrepasen la capacidad del rumen para mantener un $\mathrm{pH}$ adecuado, el cual asegure proporciones saludables en la población microbial del rumen de los animales productivos.

Esto se debe a que el pH ruminal tiende a ser más bajo cuando se alimenta con dietas altas en granos y más alto cuando las dietas tienen mayores proporciones de forraje, el cual estimula la liberación de grandes cantidades de saliva generada como producto de la masticación (NRC, 2001).

Las dietas altas en granos producen mayor cantidad de AGV totales los cuales, por su naturaleza ácida (propionato $\mathrm{pK}_{\mathrm{a}}=4,87$; acetato $\mathrm{pK}_{\mathrm{a}}=4,76$; butirato $\mathrm{pK}_{\mathrm{a}}=4,82$; lactato $\left.\mathrm{pK}_{\mathrm{a}}=3,86\right)(\mathrm{NRC}, 2001)$ han sido relacionados al desarrollo de acidosis ruminal (Oetzel, et al. 1999), enfermedad metabólica asociada a su vez con podo dermatitis aséptica difusa (también conocida como laminitis) (Manson y Leaver, 1988) y depresión de la grasa láctea (Sutton, 1989).

La adición excesiva de alimento concentrado en la dieta podría tener un efecto de depresión en la proporción de grasa láctea y por ende en la proporción de sólidos totales en la leche, debido a que al suplementar con alimento concentrado, se genera un efecto sustitutivo del forraje. Este efecto induce a una disminución de: masticación, producción de saliva, cantidad de amortiguador (buffer) que ingresa al rumen, pH ruminal y población 
de bacterias celulolíticas ruminales. Bacterias que juegan un rol importante en la síntesis de acetato, el cual es un precursor de grasa en leche (Rojas, 1995).

La disminución en la proporción de sólidos lácteos al aumentar la inclusión de alimentos concentrados en la dieta ocurre parcialmente por un efecto de dilución, pero difiere de la producción total de sólidos lácteos, la cual se favorece, debido a que se incrementa la producción total de AGV (Rojas, 1995; Sutton et al. 2003).

El efecto observado del nivel de inclusión de alimento concentrado en la dieta de las vacas sobre la proporción de sólidos totales, fue apenas una tendencia $(R 2=0,1)$. Que indica que únicamente un $10 \%$ de la variabilidad asociada al contenido de sólidos se debe a este factor. Las ecuaciones [5] y [6] (Figura 3) describen la relación entre la cantidad de alimento concentrado ofertado y la respuesta en proporción de sólidos totales lácteos.

Esta baja relación observada entre la cantidad de alimento concentrado ofertado y la proporción de sólidos totales de la población de estudio se puede explicar porque existen múltiples factores que también influyen sobre la proporción de sólidos totales producidos, tales como: raza, disponibilidad y calidad de pasto, producción de leche, etapa de lactancia, cambios en condición corporal, fraccionamiento de la ración, tamaño de partícula del forraje, entre otros (Saborío-Montero, 2011).
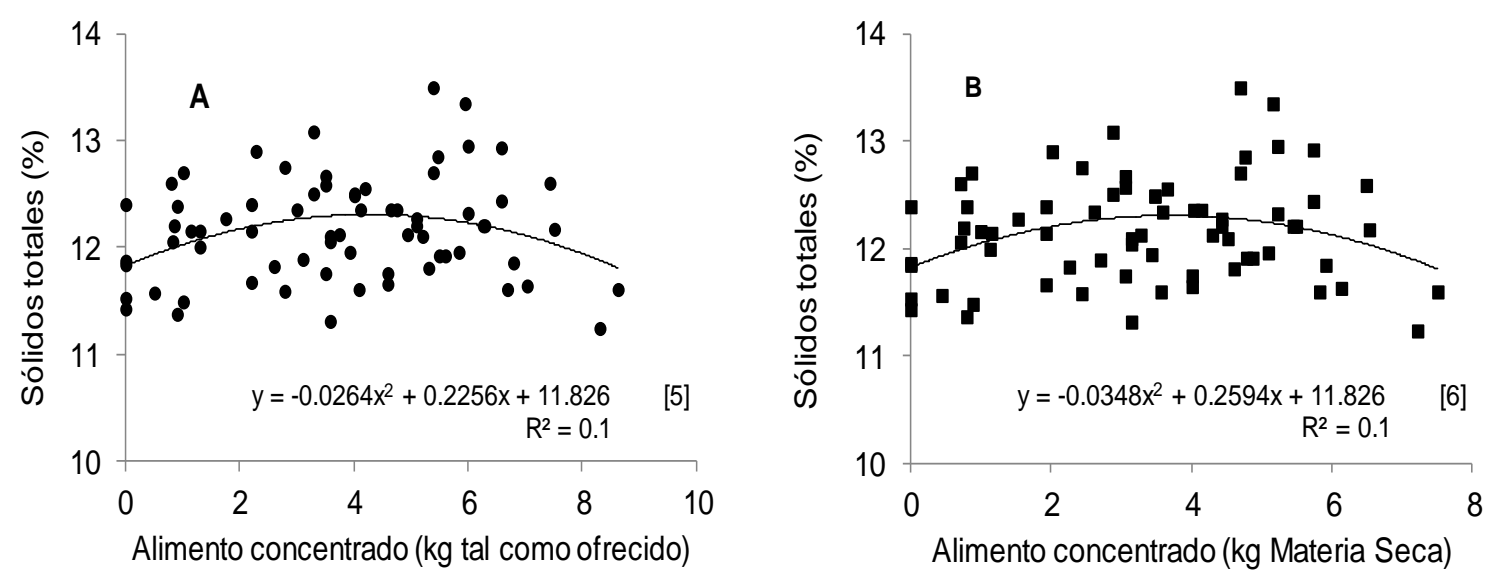

Figura 3. Efecto de la oferta de alimento concentrado tal como ofrecido $(A)$ y en materia seca (B) sobre la proporción de sólidos totales en la leche de 70 fincas de productores asociados a Productores de Monteverde S.A. 


\section{Estimación económica}

Debido a que la relación entre la oferta de alimento concentrado y la proporción de sólidos totales en la leche fue tan baja $\left(R^{2}=0,1\right)$, para efectos de calcular la ganancia neta por animal, solamente se ajustó la respuesta en producción de leche en función al efecto del alimento concentrado (Cuadro 1).

En el Cuadro 1 se evidencia que a pesar del aumento en la inversión para el rubro de alimento concentrado, el pago de leche y la ganancia neta aumentan de manera lineal conforme se incrementa el uso de este alimento, por lo tanto se puede afirmar que en las población de fincas evaluadas, es rentable utilizar hasta $\approx 9 \mathrm{~kg}$ de alimento concentrado. El comportamiento observado para producción de leche fue lineal hasta un valor máximo de inclusión de alimento concentrado tal como ofrecido de 8,6 kg/vaca/día, sin embargo es importante destacar que esta tendencia podría no ser sostenible a mayores niveles de inclusión, puesto que la producción tenderá a estabilizarse, e incluso a disminuir si se compromete la salud ruminal por un desbalance en la proporción forraje: concentrado.

Cuadro 1. Proyección de la producción de leche, inversión en concentrado, pago de leche y ganancia neta por vaca por día según dosificación de alimento concentrado en fincas asociadas a Productores de Monteverde S.A. 2007-2008.

\begin{tabular}{|c|c|c|c|c|}
\hline $\begin{array}{l}\text { Concentrado } \\
\text { ofertado } \\
\text { (kg/vaca/día) }\end{array}$ & $\begin{array}{l}\text { Leche producida } \\
\text { (kg/vaca/día) }\end{array}$ & 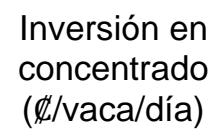 & $\begin{array}{l}\text { Pago de leche* } \\
\text { (\&//vaca/día) }\end{array}$ & 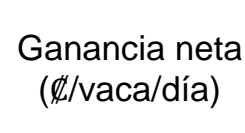 \\
\hline 0 & 5,5 & 0 & 1149 & 1149 \\
\hline 1 & 7,2 & 204 & 1505 & 1301 \\
\hline 2 & 8,8 & 408 & 1839 & 1431 \\
\hline 3 & 10,5 & 612 & 2194 & 1582 \\
\hline 4 & 12,2 & 816 & 2550 & 1734 \\
\hline 5 & 13,8 & 1020 & 2884 & 1864 \\
\hline 6 & 15,5 & 1224 & 3239 & 2015 \\
\hline 7 & 17,2 & 1428 & 3595 & 2167 \\
\hline 8 & 18,8 & 1632 & 3929 & 2297 \\
\hline 9 & 20,5 & 1836 & 4284 & 2448 \\
\hline
\end{tabular}

* Precio estimado para leche con $12,57 \%$ de sólidos totales al momento del estudio.

Tipo de cambio del dólar al momento del estudio \$491,64 por dólar (BCCR).

Nutrición Animal Tropical 9(2): 49-64. ISSN: 2215-3527/ 2015 
La información contenida en este documento puede ser utilizada como insumo para complementar la información necesaria para realizar una dosificación de alimento concentrado apropiada para cada sistema productivo, mediante la recomendación de un Ingeniero Agrónomo Zootecnista o profesional en Nutrición Animal.

\section{CONSIDERACIONES FINALES}

De este estudio se extrae que la producción promedio a base de pasto y reservas corporales para la población de estudio ronda los 5,5 kg. En promedio se produjeron1,67 $\mathrm{kg}$ de leche extra por cada $\mathrm{kg}$ de alimento concentrado que se le ofreció a la vaca, hasta cerca de $9 \mathrm{~kg}$ de alimento concentrado ofertado, valor que indica una relación de $E N_{L}$ aproximada de 0,93 Mcal en base a alimento concentrado por cada kilogramo de leche producido. Lo que hace presumir que las condiciones ambientales en los sistemas de pastoreo a las que están expuestos los animales, disminuyen la eficiencia de la utilización de la energía para conversión en leche con respecto a animales en condiciones de estabulación en países de clima templado. Sin embargo aún bajo estas circunstancias, sigue siendo rentable el uso de alimentos concentrados, con mayores beneficios económicos para aquellos sistemas que utilizan cantidades cercanas a $9 \mathrm{~kg} / \mathrm{animal} / \mathrm{día}$ en promedio.

\section{AGRADECIMIENTOS}

Los autores agradecen a los productores de leche propietarios de las fincas, a la empresa Productores de Monteverde S.A. por las facilidades brindadas en el desarrollo de esta investigación, a José Luis Vargas Leitón, Gerente General de Productores de Monteverde S.A. por el apoyo brindado y al M. Sc. Luis Arturo Villegas Zamora, al Ph. D. Jorge Alberto Elizondo Salazar y al M. Sc. Augusto Rojas Bourrillón por sus recomendaciones en el desarrollo de este documento. 


\section{LITERATURA CITADA}

Agricultural Research Council (ARC). 1980. The nutrient requirements of ruminantlivestock. Technical Review.FarnhamRoyal U.K., Commonwealth AgriculturalResearch Bureau.

Aschenbach, J.R., Penner, G.B., Stumpff, F., Gäbel, G. 2011. Ruminant nutrition symposium: Role of fermentation acid absorption in the regulation of ruminal $\mathrm{pH}$. J. Anim. Sci. 89: 1092-1107.

Balzarini, M.G., González, L., Tablada, M., Casanoves, F., Di Rienzo, J.A., Robledo, C.W. 2008. Manual del usuario. Editorial Brujas, Córdoba, Argentina.

Bargo, F.,Muller, L. D.,Delahoy, J. E.,Cassidy, T. W. 2002.Milk response to concentrate supplementation of high producing dairy cows grazing at two pasture allowances. J. DairySci. 85:1777-1792.

Bargo, F., Muller, L. D., Kolver, E. S., Delahoy, J. E. 2003. Invited Review: Production and digestion of supplemented dairy cows on pasture. J. Dairy Sci. 86: 1-42.

Delaby, L., Peyraud, J. L.,Delagarde,R. 2001. Effect of the level of concentrate supplementation, herbage allowance and milk yield at turn-out on the performance of dairy cows in mid lactation at grazing. Anim. Sci. 73:171-181.

Di Rienzo, J. A., Casanoves, F., Balzarini, M.G., González, L., Tablada, M., Robledo, C.W. 2011. InfoStat versión 2011. Grupo InfoStat, FCA, Universidad Nacional de Córdoba, Argentina. IN: http://www.infostat.com.ar

Gibb, M. J., Huckle, C. A., Nuthall, R. 2002. Effects of level of concentrate supplementation on grazing behaviour and performance by lactating dairy cows grazing continuously stocked grass swards. Anim. Sci. 74:319-335.

Hoshmand, R. Experimental research design and analysis: a practical approach for agricultural and natural sciences. 1994. CRC Press. 408pp.

Kaps, M., Lamberson, W. 2009. Biostatistics for animal science. An introductory text. $2^{\text {nd }}$ Edition, CABI International. 504pp. 
Manson, F.J., Leaver, J.D. 1988. The influence of concentrate amount on locomotion and clinical lameness in dairy cattle. Anim. Prod. 47:185-190.

Meyeres, P., Stoll, J., Bormann, J., Reents, R., Gengler, N., 2004. Prediction of daily milk, fat and protein production by a random regression test-day model. J. Dairy Sci. 87:1925-1933.

National Research Council. 2001. Nutrient Requirements of Dairy Cattle. 7th rev. ed. National Academy Press. Washington, D.C. 381pp.

Noordhuizen J.P.T.M., Buurman J. 1984. Veterinary automated management and production control program for dairy farms (VAMPP). The application of MUMPS for data processing. Vet Q. 6:2, 66-72.

Oetzel, G.R., Nordlund, K.V., Garrett, E.F.1999. Effect of ruminal pH and stage of lactation on ruminal lactate concentrations in dairy cows. J. Dairy Sci. 82(Suppl. 1):38.

Reis, R. B., Combs, D. K. 2000. Effects of increasing levels of grain supplementation on rumen environment and lactation performance of dairy cows grazing grasslegume pasture. J. Dairy Sci. 83:2888-2898.

Robaina, A. C., Grainger, C.Moate, P. Taylor, J.Stewart, J. 1998. Responses to grain feeding by grazing dairy cows. Aust. J. Exp. Agric. 38:541-549.

Rojas, A. 1995. Conceptos básicos en nutrición de rumiantes. Escuela de Zootecnia. Facultad de Agronomía. Universidad de Costa Rica. 178 pp.

Saborío-Montero, A. 2010. Utilización de sistemas de posicionamiento global (GPS) en la estructuración de apartos para pastoreo. Revista ECAG Informa. 54: 54-58.

Saborío-Montero, A. 2011. Factores que influencian el porcentaje de sólidos totales de la leche. Revista ECAG Informa. 56: 70-73.

Sánchez, J., Soto, H. 1999. Estimación de la calidad nutricional de los forrajes del cantón de San Carlos III. Energía para la producción de leche. Nutrición Animal Tropical. Vol. 5, Núm. 1.

Sayers, H. J. 1999. The effect of sward characteristics and level and type of supplement on grazing behaviour, herbage intake and performance of lactating dairy cows. 
Ph.D. Thesis. Queen's University of Belfast. The Agricultural Research Institute of Northern Ireland. Hillsborough.

Storm, A.C., Kristensen, N.B., Hanigan M.D. 2012.A model of ruminal volatile fatty acid absorption kinetics and rumen epithelial blood flow in lactating Holstein cows. J. Dairy Sci. 95: 2919-2934.

Storm, A.C., Kristensen, N.B. 2010.Effect of particle size and dry matter content of a total mixed ratio on intraruminal equilibration and net portal flux of volatile fatty acids in lactating dairy cows. J. Dairy Sci. 93: 4223-4238.8

Sutton, J.D. 1989. Altering milk composition by feeding. J. Dairy Sci. 72:2801-2814.

Sutton, J.D., Dhanoa, M.S., Morant, S.V., France, J., Napper, D.J., Schullen, E. 2003. Rates of production of acetate, propionate, and butyrate in the rumen of lactating dairy cows given normal and low-roughage diets. J. Dairy Sci. 86: 3620-3633.

Tyrrell, H.F., Reid, J.T. 1965. Prediction of the energy value of cow's milk. J. DairySci. 48:1215-1223.

Villalobos, L., Arce, J. 2014. Evaluación agronómica y nutricional del pasto estrella africana (Cynodon nlemfuensis) en la zona de Monteverde, Puntarenas, Costa Rica. II. Valor Nutricional. Agronomía Costarricense. 38(1):133-145.

Walker, G. P., Stockdale, C. R.,Wales, W. J., Doyle, P. T., Dellow, D. W. 2001. Effect of level of grain supplementation on milk production responses of dairy cows in midlate lactation when grazing irrigated pastures high in paspalum (Paspalum dilatatum Poir.). Aust. J. Exp. Agric. 41:1-11. 